Generalized Contraflow for Evacuation Planning Problem

\title{
Generalized Contraflow for Evacuation Planning Problem
}

\author{
Urmila Pyakurel \\ Central Department of Mathematics, Tribhuvan University, P.O.Box 13143, Kathmandu, Nepal \\ Correspondence to: Urmila Pyakurel, email:urmilapyakurel@gmail.com
}

\begin{abstract}
The research in evacuation planning has been very much motivated due to the rapidly increased number of disasters world wide. It supports to remove the evacuees from dangerous areas to safe areas. Contraflow reconfiguration during evacuation make traffic smooth by reversing the required road directions from dangerous areas to safe areas that improve both flow and speed significantly. On lossy network, the generalized maximum dynamic contraflow and generalized earliest arrival contraflow problems have been solved efficiently with pseudo-polynomial time.

This paper focuses in analytical solutions on generalized contraflow for evacuation planning problem. The problems are considered on two terminal lossy networks. We solve the generalized maximum static contraflow problem in pseudo-polynomial time and compute its approximation solution in polynomial time. Moreover, we present a fully polynomial time approximation scheme (FPTAS) to compute an approximate generalized maximum dynamic contraflow solution.
\end{abstract}

Keywords: Evacuation planning, Lossy network, Generalized contraflow, Approximation scheme.

\section{Introduction}

The importance of evacuation planning is rising day by day due to the increasing number of large scale disasters world-wide like hurricanes, earthquakes, tsunamis, flooding, landslide, industrial and nuclear accidents, fire, terrorist attacks and wars. The most cited examples are the Haiti, Chile, Chichi, Bam and Kashmir earthquakes, the tsunami in the Indian Ocean and Japan, the major hurricanes Katrina, Rita and Sandy, and the September 11 attacks in New York City and Washington D.C. As a recent example, Nepal Earthquake of magnitude 7.8 Richter scale on 25 April 2015 and two powerful aftershocks killed at least 9000 people and destroyed more than 600,000 houses. After any kind of disasters, the problem of saving evacuees and normalizing the situation is challenging. Among many others, an effective evacuation solution has been lacked without a prior evacuation planning. A case study has been carried out before this disaster [21], but is still applicable as scientists have warned that even larger magnitude quake could rock Kathmandu and the Himalaya Frontal Fault.

The very challenging emergency issues because of large scale disasters promote the research on evacuation planning. An evacuation planning problem is understood as the procedure of shifting evacuees from disastrous areas to safety areas as successfully as possible with minimum lost of lives and properties. The evacuation network is defined as a network that corresponds to a region to be evacuated in which the intersections of streets including the terminal places represent the nodes and the connections between these parts denote the edges. The initial locations where evacuees can gather are the source nodes and the locations at safety regions are the sink nodes. Each arc has transit time or cost, capacity and loss factor. The group of evacuees that passes through the network over time is modeled as a flow.

We can find various mathematical models that have been developed for flow maximization and time minimization in quite diversified research domains from engineering, mathematical, management and social sciences. We refer to [2] and the references therein for details. The flow maximization model maximizes the flow receiving at safe areas in given time. However, the given time itself is in question. These models has been generalized by considering the loss or gain factors which deal with the issues where real flow may be in lesser or greater amount on every arc due to unfortunate death of evacuees 13 . This generalized flow model has crucial role in more realistic evacuation planning scenarios.

In evacuation planning, contraflow is a very useful and widely accepted model. It increases the outbound road capacities by reversing the direction of arcs from the sources to the sinks. It minimizes the congestion and makes the traffic systematic and smooth. It is emerging to react to different large scale natural and man-made disasters. However, it is a very challenging issue of finding a network reconfiguration with 
ideal lane directions satisfying the given constraints to optimize the given objective function. Authors in [9] proved that the evacuation time is reduced at least 40 percent with at most 30 percent of the total arc reversals from the practical point of view. Various mathematical models, heuristics, optimization and simulation techniques for the network with contraflow approach have been discussed in [2].

Most of the earlier works on contraflow focus on the heuristic techniques, however the analytical approach has been considered quite recently. The flow values obtained by contraflow models increase significantly that may be doubled for given time horizon. In fact, the contraflow solutions are at most two times faster than the solutions without contraflow to transship the given flow value from the sources to the sinks. Some contraflow problems with efficient analytical solution algorithms in different particular networks have been presented in [3, 15, 16, 17, 22. Some special structured network dynamic flow efficient solutions with continuous time settings on the arcs can be found in [4, 18, 19, 20,

Authors in [14] have introduced the generalized contraflow model and solved the generalized maximum dynamic contraflow (GMDCF) problem on two terminal lossy network. If the loss factor is $\leq 1$ for all arcs on a network, then it is called lossy network. Any network without flow-generating cycles can be turned into lossy networks. The GMDCF solution sends the maximum flow value to the sink through the minimum loss factors in given time horizon $T$. They have also solved the generalized earliest arrival contraflow (GEACF) problem that sends maximum flow to the sink at every time steps $t, 0 \leq t \leq T$ with minimum loss factors. Both problems GMDCF and GEACF are solved in strongly polynomial time complexity in the size of time expanded network.

In this paper, we study the static nature of the generalized contraflow approaches as a foundation on two terminal lossy networks. However, it is solved in pseudo-polynomial time complexity. We also compute its approximation solutions in polynomial time complexity. Moreover, we present an FPTAS to compute an approximate solution for the GMDCF problem on two terminal lossy networks.

The paper is organized as follows. In Section 2, we model the generalized contraflow evacuation planning problem. The generalized static contraflow problem on two-terminal lossy network is presented in Section 3 With quick look on the problems GMDCF and GEACF, we present an approximation result for the GMDCF problem on two terminal lossy networks in Section 4. Concluding remarks with open problem conjectures are given in Section 5

\section{Mathematical Models}

An evacuation scenario is represented by a network $\mathcal{N}=(V, A)$, where $|V|=n$ and $|A|=m$ with a set of nodes $V$ and a set of $\operatorname{arcs} A$. Here, the network represents a region to be evacuated where road intersections are the nodes and connections between them are the arcs. Initial locations, where evacuees can gather are the source nodes and the locations at safety regions are the sink nodes. For the contraflow scenario, two way network configuration will be allowed. Let $S$ and $D$ be the sets of sources and sinks for a multi terminal network, respectively. For a two terminal network, let $S=\{s\}$ and $D=\{d\}$. We assume that $A_{d}^{\text {out }}=A_{s}^{\text {in }}=\emptyset$, where $A_{v}^{\text {out }}=\{(v, w) \in A\}$ and $A_{v}^{\text {in }}=\{(w, v) \in A\}$ for $v \in V$. Other evacuation scenarios, like building or aircraft, do have similar interpretations.

The group of evacuees that passes through the network over time is modeled as a flow in a dynamic network. Each arc has integer capacity $b_{A}(e)$ that gives the upper bound of flow that may enter the node $v$ of $e=(v, w) \in A$ per time period. The node capacities $b_{V}(v), v \in V$ bound the amount of evacuees allowed to hold at $v$. The symmetric travel time on $e=(v, w) \in A$ is the time needed to travel one unit of flow from $v$ to $w$ and it is represented as $\tau(e)$. Each arc has the loss factor $\lambda(e) \in \mathcal{R}^{+}$. If one unit of flow enters $e=(v, w)$ from $v$ at time $t$, only $\lambda(e)$ units leave from $w$ at time $t+\tau(e)$. If $\lambda(e) \leq 1$ for all arcs $e \in A$, then the network turns into lossy network, where flow is only lost but never gained along the arcs. There are different factors that make the flow lost during evacuation process. However, we consider a special case in which we assume that in each time unit the same percentage of the remaining flow value is lost. Thus we consider $\lambda \equiv 2^{\text {c. } \tau}$ for some constant $c<0$.

The generalized dynamic transportation network $\mathcal{N}=\left(V, A, b_{A}, \tau, \lambda, S, D, T\right)$ is represented by the collection of all data in the evacuation scenario with a predetermined time $T$. We assume a finite time horizon $T$, that means everything must happen before given time $T$. It could be estimated minimum $T$, the flow would be quickest. Time can increase in discrete increments or continuously. The continuous time gives the accurate solution, however, it takes long time to give solution. So, we consider the discrete time 
units $t=0,1, \ldots, T$ and all time related parameters are assumed to integers. The choice of time unit affects the problem directly, i.e., if the time unit is shorter then the problem is more complex. Let $\mathbf{T}$ be the domain of time, i.e., $\mathbf{T}=\{0,1, \ldots, T\}$. If the scenario is static, i.e., the evacuation is possible in a single wave, then the generalized network is represented as $\mathcal{N}=\left(V, A, b_{A}, c_{A}, \lambda, S, D\right)$, where $c_{A}$ is the integer cost needed for sending one unit of evacuees through the arc $A$. Here, the same terminology "network" is used for both type of networks.

Let the reversal of $e=(v, w) \in A$ be denoted by the arc $e^{-1}=(w, v)$. For a given generalized network $\mathcal{N}=\left(V, A, b_{A}, \tau, \lambda, S, D, T\right)$ with integer inputs and symmetric travel times and loss factors, the generalized auxiliary network $\overline{\mathcal{N}}=\left(V, E, b_{E}, \tau, \lambda, S, D, T\right)$ consists of the modified arc capacities and travel times, for all $\bar{e} \in E$ as follows:

$$
b_{E}(\bar{e})=b_{A}(e)+b_{A}\left(e^{-1}\right) \text { and } \tau(\bar{e})=\left\{\begin{array}{l}
\tau(e) \text { if } e \in A \\
\tau\left(e^{-1}\right) \text { otherwise }
\end{array}\right.
$$

where, an edge $\bar{e} \in E$ in $\overline{\mathcal{N}}$ if $e \vee e^{-1} \in A$ in $\mathcal{N}$. The remaining graph structure and the data are unaltered. The modified loss factor is $\lambda(\bar{e})$ with $\lambda(\bar{e}) \equiv 2^{c . \tau(\bar{e})}, c<0$.

For a static network $\mathcal{N}=\left(V, A, b_{A}, \lambda, S, D\right)$, the generalized auxiliary network $\overline{\mathcal{N}}=\left(V, E, b_{E}, \lambda, S, D\right)$ has an analogous representation discarding the time factors.

For a two terminal network $\mathcal{N}=\left(V, A, b_{A}, \tau, \lambda, s, d, T\right)$, the non-negative function $x_{d y n a}: A \times \mathbf{T} \rightarrow \mathcal{R}^{+}$ represents the generalized dynamic flow (GDF). A generalized dynamic $s$ - $d$ flow $x_{d y n a}$ for given time $T$ satisfies Constraints (1-3). Inequality (2) allows wait to flow at intermediate nodes, otherwise equality holds.

$$
\begin{aligned}
\sum_{\sigma=\tau(e)}^{T} \sum_{e \in A_{v}^{i n}} \lambda(e) x_{d y n a}(e, \sigma-\tau(e)) & =\sum_{\sigma=0}^{T} \sum_{e \in A_{v}^{\text {out }}} x_{d y n a}(e, \sigma), v \neq s, d \\
\sum_{\sigma=\tau(e)}^{t} \sum_{e \in A_{v}^{i n}} \lambda(e) x_{d y n a}(e, \sigma-\tau(e)) & \geq \sum_{\sigma=0}^{t} \sum_{e \in A_{v}^{\text {out }}} x_{d y n a}(e, \sigma), v \neq s, t \in \mathbf{T} \\
0 \leq \lambda(e) x_{\text {dyna }}(e, t) & \leq b_{A}(e, t)
\end{aligned}
$$

To ensure that no flow remains in the network at time $T$, we assume that $x_{d y n a}(e, t)=0$ for all $t \geq T-\tau(e)$. The flow value $\operatorname{val}\left(x_{d y n a}, T\right)$ in Equation (4) is the amount of GDF $x_{d y n a}$ sent to the sink within $T$. The generalized maximum dynamic flow (GMDF) problem is to find a GDF of maximum value $\operatorname{val}\left(x_{d y n a}, T\right)$.

$$
\operatorname{val}\left(x_{d y n a}, T\right)=\sum_{e \in A_{d}^{i n}} \sum_{\sigma=\tau(e)}^{T} \lambda(e) x_{d y n a}(e, \sigma-\tau(e))
$$

The total amount of flow that arrives at the sink in time steps $\{0, \ldots, t\}$ for all $t \in \mathbf{T}$ gives the arrival pattern of a GDF. Authors in [7, 8] studied the GMDF problem.

Analogously, if the network is generalized static $\mathcal{N}=\left(V, A, b_{A}, \lambda, s, d\right)$, then a non-negative function $x_{\text {stat }}: A \rightarrow \mathcal{R}^{+}$represents a generalized static flow (GSF). The flow value of GSF $x_{\text {stat }}$ is written as $\operatorname{val}\left(x_{\text {stat }}\right)$. The generalized maximum static flow (GMSF) has been studied in [11, 12.

The generalized maximum dynamic contraflow (GMDCF) model is defined on generalized evacuation network with objective Function (4) satisfying the Constraints (1-3) and having arc reversal capability on the network. Analogously, the generalized maximum static contraflow (GMSCF)model is defined by discarding the time factor.

We define the generalized residual network as $\mathcal{N}^{R}\left(x_{\text {stat }}\right)=(V, \vec{A} \cup \overleftarrow{A})$ of $\mathcal{N}=\left(V, A, b_{A}, \tau, \lambda, s, d, T\right)$ where $\vec{A}=\left\{\vec{e}=e \mid x_{\text {stat }}(e)<b_{A}(e)\right\}$ be the set of forward arcs with capacity $b_{A}(e)-x_{\text {stat }}(e)$, transit time $\tau(e)$ and loss factor $\lambda(e)$, and $\overleftarrow{A}=\left\{\overleftarrow{e}=(\operatorname{head}(e)\right.$, tail $\left.(e)) \mid x_{\text {stat }}(e)>0\right\}$ be the set of backward arcs with capacity $\frac{1}{\lambda(e)} x_{\text {stat }}(e)$, a transit time $-\tau(e)$ and loss factor $\frac{1}{\lambda(e)}$.

For a generalized network $\mathcal{N}$, the generalized time expanded network $\mathcal{N}(T)=\left(V_{T}, A_{M} \cup A_{H}\right)$ is defined as an expansion of the dynamic network where each node $v$ of the static graph is copied $T$ times to obtain 
a node $v(t)$ for each $v \in V$ and each $t \in\{0, \ldots, T\}$. For each $\operatorname{arc} e=(v, w) \in A$, the arc from $v(t)$ to $w(t+\tau(v, w))$ with capacity $b_{A}(v, w), \forall(v, w) \in A_{M}$ and loss factor $\lambda(v, w), \forall(v, w) \in A_{M}$ is called movement arc. For each $\operatorname{arc} e=(v, w) \in A$, the arc from $v(t)$ to $v(t+1)$ with capacity $b_{A}(v, v)=$ $\infty, \forall(v, v) \in A_{H}$ and loss factor $\lambda(v, v)=1, \forall(v, v) \in A_{H}$ is called holdover arc which allows storage at the nodes.

Let $P_{k}$ and $C_{k}$, be a path and a cycle. The time needed to cross $P_{k}$ and $C_{k}$, are defined as $\tau\left(P_{k}\right)=$ $\sum_{e \in P_{k}} \tau(e)$ and $\tau\left(C_{k}\right)=\sum_{e \in C_{k}} \tau(e)$. Similarly, the loss factors for a path $P_{k}$ and a cycle $C_{k}$ are defined as $\lambda\left(P_{k}\right)=\prod_{e \in P_{k}} \lambda(e)$ and $\lambda\left(C_{k}\right)=\prod_{e \in C_{k}} \lambda(e)$. Cycles $C_{k}$ with $\lambda\left(C_{k}\right)=1, \lambda\left(C_{k}\right)>1$ and $\lambda\left(C_{k}\right)<1$ are known as unit loss cycles, flow generating cycles and flow absorbing cycles, respectively.

\section{Generalized Static Contraflow Problem}

In generalized network, if every arc has a capacity, cost and a symmetric loss factor then it turns into generalized static network. We present the GMSCF problem and give an efficient algorithm to compute its solution in Subsection 3.1. However, the GMSCF solution is not obtained in polynomial time complexity. Thus we present polynomial time algorithms to compute an approximate GMSCF solution in Subsection 3.2

\subsection{Generalized maximum static contraflow}

Problem 1. Given a generalized network $\mathcal{N}=\left(V, A, b_{A}, \lambda, s, d\right)$, the GMSCF problem is to find the maximum amount of flow that can be sent from $s$ to $d$ if the direction of arcs can be reversed at time zero.

To solve Problem 1 we develop an efficient algorithm. Our algorithm is based on the MSCF algorithm of [1, 22] for the MSCF problem and the successive shortest path algorithm (SSPA) introduced in [11, 12. for the GMSF problem.

Algorithm 1. Generalized maximum static contraflow algorithm

1. Input: Given generalized network $\mathcal{N}=\left(V, A, b_{A}, c_{A}, \lambda, s, d\right)$

2. Construct the generalized auxiliary network $\overline{\mathcal{N}}=\left(V, E, b_{E}, c_{E}, \lambda, s, d\right)$

3. On $\overline{\mathcal{N}}$, generate a solution for MSCF using the SSPA of [11, [12] with capacity $b_{E}(\bar{e})$, and loss factor $\lambda(\bar{e})$.

4. Based on the solution in Step 3, arc $(w, v) \in A$ is reversed, if and only if the flow along arc $(v, w)$ is greater than $b_{A}(v, w)$ or if there is a non-negative flow along arc $(v, w) \notin A$.

5. Output: a GMSCF solution on two terminal lossy network $\mathcal{N}$.

The SSPA of [11, 12 on auxiliary network $\overline{\mathcal{N}}$ with reversed capacity $b_{E}(\bar{e})$ and loss factor $\lambda(\bar{e})$ works as follows. It starts with $x_{\text {stat }}=0$ and the residual network $\overline{\mathcal{N}}^{R}$. If there is no $s-d$ path in $\overline{\mathcal{N}}^{R}$, the algorithm terminates. Otherwise, it augments flow along a $s-d$ path of minimum loss. The process will be continued with the resulting $x_{\text {stat }}$ flow and residual network $\overline{\mathcal{N}}^{R}$. Moreover, if the network has no residual flow generating cycle, then only, the obtained flow is a GMSF in $\overline{\mathcal{N}}$ [11, 12]. The SSPA has pseudo-polynomial time complexity.

A contraflow solution of static network is equivalent to the solution without contraflow on the corresponding auxiliary network [1, 22. Standing on the result of Onaga [11, 12, we prove Lemma 3.1] that gives the optimality criteria for the GMSCF problem.

Lemma 3.1. A generalized $s-d$ flow in $\overline{\mathcal{N}}$ is a GMSCF solution in $\mathcal{N}$ if there is neither a $s-d$ path along which flow can be augmented nor a flow generating cycle connected to the sink in residual network of $\overline{\mathcal{N}}$.

Proof. The generalized static network $\mathcal{N}=\left(V, A, b_{A}, c_{A}, \lambda, s, d\right)$ is converted into generalized auxiliary network $\overline{\mathcal{N}}=\left(V, E, b_{E}, c_{E}, \lambda, s, d\right)$ by contraflow reconfiguration. On the generalized auxiliary network $\overline{\mathcal{N}}$, we augment flow successively from the source $s$ to the sink $d$ until there exists an augmenting path in the 
corresponding residual network $\overline{\mathcal{N}}^{R}$. As we consider the lossy network, there will not exist flow generating cycle. If there is no augmenting path on the auxiliary residual network, the GMSF is computed in the generalized auxiliary network $\overline{\mathcal{N}}$. A GMSF solution in $\overline{\mathcal{N}}$ is equivalent to the GMSCF solution in $\mathcal{N}$.

Algorithm 1 is feasible. Steps 2 and 3 are feasible. As the successive shortest paths have no any cycles, there is either a flow along arc $(v, w)$ or $(w, v)$ but never in both arcs, so Step 4 is also feasible. Now, Theorem 3.2 proves its optimality.

Theorem 3.2. Algorithm 1] finds an optimal solution to Problem 1 on any two terminal lossy network $\mathcal{N}$.

Proof. We transform the network $\mathcal{N}$ for Problem 1 into the generalized auxiliary network $\overline{\mathcal{N}}$. Recall that the arc capacity is increased by adding the capacities of both directions between the nodes but the loss factor remains the same. An optimal solution to the GMSF problem on $\overline{\mathcal{N}}$ could be obtained by solving it iteratively using the SSPA of [11, 12. However, at each iteration, a GMSF solution on $\overline{\mathcal{N}}$ is equivalent to the GMSCF solution on $\mathcal{N}$. Therefore, an optimal solution for the GMSCF problem can be solved by solving the GMSF problem on $\overline{\mathcal{N}}$.

Corollary 1. The running time of Algorithm 1 is pseudo-polynomial.

Example 1. Each arc of the evacuation network $\mathcal{N}$ has capacity and loss factor as shown in Figure 1 (a). We construct the contraflow reconfiguration network as shown in Figures $11(b)-1($ (c)) where the capacity of each arc increases but the loss factor remains same. Now we consider the successive shortest paths on $\overline{\mathcal{N}}$ as follows:

$$
\begin{aligned}
& P_{1}=s-X-d \quad \text { with } \lambda\left(P_{1}\right)=\prod_{e \in P_{1}} \lambda(e)=\frac{1}{3} \times \frac{1}{2}=\frac{1}{6} \\
& P_{2}=s-X-Y-d \quad \text { with } \lambda\left(P_{2}\right)=\prod_{e \in P_{2}} \lambda(e)=\frac{1}{3} \times \frac{2}{3} \times \frac{4}{5}=\frac{8}{45} \\
& P_{3}=s-Y-X-d \quad \text { with } \lambda\left(P_{3}\right)=\prod_{e \in P_{3}} \lambda(e)=\frac{1}{2} \times \frac{2}{3} \times \frac{1}{2}=\frac{1}{6} \\
& P_{4}=s-Y-d \quad \text { with } \lambda\left(P_{4}\right)=\prod_{e \in P_{4}} \lambda(e)=\frac{1}{2} \times \frac{4}{5}=\frac{2}{5}
\end{aligned}
$$

Since, $\lambda\left(P_{1}\right)=\lambda\left(P_{3}\right)<\lambda\left(P_{2}\right)<\lambda\left(P_{4}\right)$, the paths $P_{1}$ and $P_{3}$ are the paths with minimum loss factors according to SSPA of Onaga [11, 12] as shown in Figure 1](d) that gives a GMSF solution. This GMSF solution is equivalent to a GMSCF solution on the original network $\mathcal{N}$.
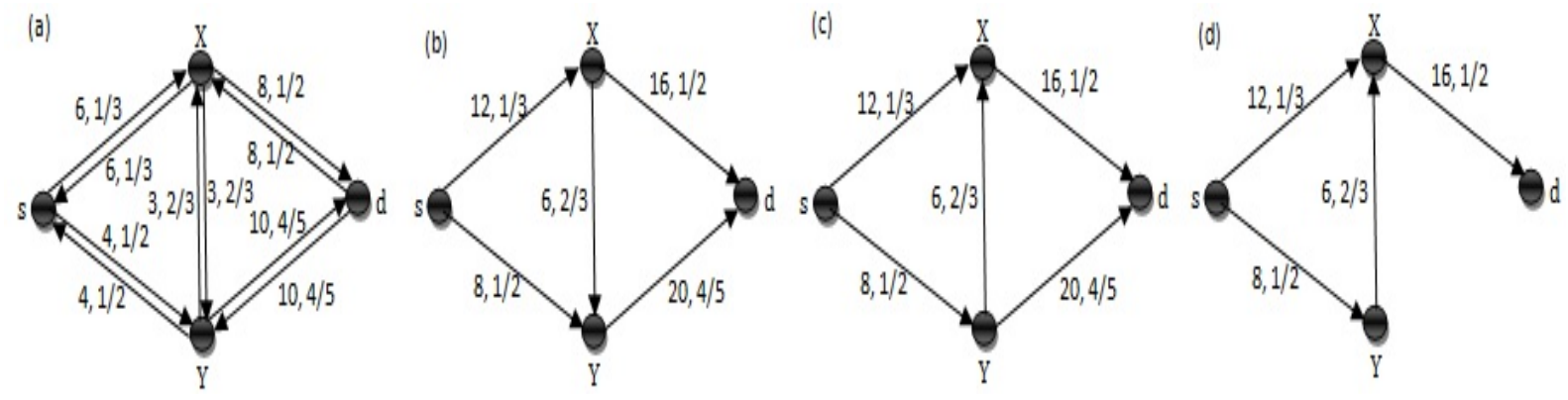

Figure 1: (a) $\mathcal{N}$-evacuation scenario, (b)-(c) $\overline{\mathcal{N}}$-contraflow configuration, (d) $\overline{\mathcal{N}^{\prime}}$ - minimum loss network

\subsection{Approximate generalized maximum static contraflow}

For the GMSCF problem, it is still unknown whether a strongly polynomial time algorithm exists. It is due to the fact that there is no any polynomial time algorithm without contraflow model. In this section, we introduce an approximate GMSCF problem and design approximation algorithms for it. 
We consider a two terminal lossy network without flow generating cycles in which the product of gain factors exceeds one. Note that a lossy network cannot have flow generating cycles and networks with no flow generating cycles need not be lossy. However, the transformation of a network with no flow generating cycles into an equivalent lossy networks has been made in $O(m n)$ time $[5]$.

We present an FPTAS based on the packing algorithm of 5 that computes a $\epsilon$-approximate GMSCF solution on two-terminal lossy network without flow generating cycles for given $\epsilon>0$. We also discuss whether the propose FPTAS works on network with flow generating cycles.

Problem 2. Given a generalized network $\mathcal{N}=\left(V, A, b_{A}, c_{A}, \lambda, s, d\right)$, non-negative cost, symmetric loss and $\epsilon>0$, does there exist an $\epsilon$-approximate GMSCF solution with value at least $\epsilon$-optimal GMSCF, if the direction of arcs can be reversed at time zero without any processing cost?

In order to solve the $\epsilon$-approximate GMSCF problem, Problem 2 in the lossy network without flow generating cycle, we present an FPTAS (cf. Algorithm 2). Our algorithm for this problem is based on MSCF algorithm of [1, 22 for MSCF problem on two terminal general network in which flow is always conserved and the generalized max flow packing algorithm of 5 for GMSF problem on the two terminal lossy network. Recall that in lossy network, flow is either conserved or lost in each arc.

Algorithm 2. Approximate generalized maximum static contraflow algorithm

1. Input: given a generalized network $\mathcal{N}=\left(V, A, b_{A}, c_{A}, \lambda, s, d\right)$, and $\epsilon>0$.

2. Construct the auxiliary lossy network $\overline{\mathcal{N}}=\left(V, E, b_{E}, c_{E}, \lambda, s, d\right)$.

3. On $\overline{\mathcal{N}}$, generate the generalized max flow solution using the packing algorithm of [5] with capacity $b_{E}(\bar{e})$, and loss factor $\lambda(\bar{e})$.

4. Based on the solution in Step 3, arc $(w, v) \in A$ is reversed, if and only if the flow along arc $(v, w)$ is greater than $b_{A}(v, w)$ or if there is a non-negative flow along arc $(v, w) \notin A$.

5. Output: an $\epsilon$-approximate GMSCF solution on lossy network $\mathcal{N}$.

To solve Problem 2, we first convert the given network into auxiliary network as in Step 2 of Algorithm 2 Then we solve the $\epsilon$-approximate GMSF problem on the auxiliary lossy network $\overline{\mathcal{N}}$ with non-negative cost using the generalized max flow packing algorithm of [5] in Step 3. The generalized max flow packing algorithm works on original network but not in residual network. If a network has special structure like no flow generating cycles or lossy network, then this property is remained throughout the algorithm. However, the residual network does not maintain such property. To solve the $\epsilon$-approximate GMSF problem on lossy network, we need to compute a generalized shortest path $P$ by solving the generalized shortest path problem. The problem is to send flow from the source $s$ so that one unit of flow arrives at the sink $d$ as cheaply as possible. The generalized shortest path, on lossy auxiliary network $\overline{\mathcal{N}}$, computes an optimal solution if the solution sends flow only along a single $s-d$ path. Authors in $[5]$ solved the generalized shortest path problem which is based on Dijkstra algorithm in the lossy network with nonnegative length in $O(m+n \log m)$ time complexity.

Let $\pi(v)$ be the cheapest cost of sending flow from $s$ to each node $v \in V$. Once the node $v$ with cheapest cost is obtained, it is deleted from the priority queue. Then the distances of all neighbors are updated. Moreover, let $\pi(v)$ be the unit cost of shifting flow at node $v$. Now the unit flow at $v$ is shifted to another node $w$ along arc $(v, w)$ with cost $\pi(v)+c_{E}(v, w)$. As each arc $(v, w)$ has loss factor, only $\lambda(v, w)$ units reach to node $w$. Thus, the scaling should be done with loss factor. The cheapest cost $\pi(w)$ for node $w$ is obtained as $\min \left\{\pi(w), \frac{\pi(v)+c_{E}(v, w)}{\lambda(v, w)}\right\}$. Continue this process until the cheapest cost $\pi(d)$ for sink $d$ is obtained. The resulting path is the generalized shortest path.

Once the generalized shortest path $P$ is obtained, the generalized max flow packing algorithm, which is based on linear programming formulation (see, 5]), sends as much flow as possible along path $P$ without violating the capacity constraints in lossy auxiliary network $\overline{\mathcal{N}}$. The generalized max flow packing algorithm terminates after $O\left(\epsilon^{-2} m \log m\right)$ iterations [5]. We state Lemma 3.3 on the lossy auxiliary network $\overline{\mathcal{N}}$ for the sake of completeness similar to the result in [5] on original network. 
Lemma 3.3. In lossy auxiliary network $\overline{\mathcal{N}}$, an $\epsilon$-approximate GMSF can be computed in $O\left(\epsilon^{-2} m(m+\right.$ $n \log m) \log m)$ time.

\section{Theorem 3.4. Algorithm 2 solves Problem 2 efficiently.}

Proof. The Algorithm 2 is feasible. Steps 2 and 3 are feasible as the generalized shortest paths have no any cycles. Since there is either a flow along arc $(v, w)$ or $(w, v)$ but never in both arcs, Step 4 is also feasible.

Now we prove its correctness. We transform the lossy network $\mathcal{N}$ for Problem 2 into the auxiliary lossy network $\overline{\mathcal{N}}$. With contraflow reconfiguration, the arc capacity is increased by adding the capacities of both directions between the nodes but the loss factor remains the same. An $\epsilon$-approximate solution to the GMSF problem on $\overline{\mathcal{N}}$ could be obtained by solving it iteratively using the generalized max flow packing algorithm of [5]. The obtained $\epsilon$-approximate GMSF solution on $\overline{\mathcal{N}}$ at each iteration is equivalent to the $\epsilon$-approximate GMSCF solution on $\mathcal{N}$ as in optimal solutions of [1, 22].

Corollary 2. An $\epsilon$-approximate GMSCF solution is computed in $O\left(\epsilon^{-2} m(m+n l o g m)\right.$ logm $)$ time complexity.

Proof. The complexity of Algorithm 2 is dominated by complexity of Step 3. As the generalized max flow packing algorithm of [5] terminates after $O\left(\epsilon^{-2} m \log m\right)$ iterations and at each iteration a generalized shortest path is computed in $O(m+n \log m)$ time. Combining these two complexities, we meet the result similar as in Lemma 3.3 .

Now we improve our approximation algorithm, Algorithm 2 so that it can solve the GMSCF problem exactly even in networks with flow-generating cycles. By assuming $\epsilon=1 / 2$ for the generalized max flow packing algorithm has been run that computes $1 / 2$-approximate GMSF $x_{\text {stat }}$ in $\overline{\mathcal{N}}$. The generalized max flow packing algorithm is repeatedly run in the corresponding residual network $\overline{\mathcal{N}}^{R}$, adding the resulting flow to $x_{\text {stat }}$. As in each iteration, at least $1 / 2$ of the remaining flow is possible, the optimality gap geometrically decreases to zero. After $\log (1 / \epsilon)$ iterations an $\epsilon$-approximation will be obtained using the generalized max flow packing algorithm of [5]. Without loss of generality, we assume that $\epsilon$ is sufficiently small, for example, say $M^{-3 m}$ where $M$ denotes the biggest integer used to represent any of the costs, capacities or loss factors. Then, the $\epsilon$-approximate flow induced by the generalized max flow packing algorithm on $\overline{\mathcal{N}}$ can be efficiently rounded to an optimal flow as in 6 .

However, this method has some difficulties in computation because the residual network may have the flow generating cycles although the original network did not have. If there is flow generating cycles then the generalized shortest path problem does not work. Before using the generalized max flow packing algorithm, we have to cancel flow generating cycles in the residual network using the canceling residual flow generating cycles algorithm of [6] in which we repeatedly send flow around flow generating cycle until at least one arc becomes saturated. That creates excess at one node of the cycle and if the cycles are chosen carefully, all the flow generating cycles can be canceled in $O\left(m n^{2} \log M\right)$ [6. However, authors in [5] improved the complexity of canceling flow generating cycle to $O(m n \log \log M)$ using the gain-scaling technique of [23]. In the process of removing flow generating cycles, the residual network may contain nodes with excess. To remove it we have to add an arc from source $s$ to the nodes with excess and make its capacity equal to the node's excess. Then only we can use the generalized max flow packing algorithm with $\epsilon=1 / 2$ in the residual network and the excess created from canceling flow generating cycles can be sent to the sink by the algorithm [5].

Theorem 3.5. An exact GMSCF can be computed on two terminal generalized network efficiently.

\section{Generalized Dynamic Contraflow Problem}

In the generalized dynamic network, we assume that the transit time and loss factor in each arc are symmetric. We also assume that we reverse the direction of arc from source to sink without any processing cost. We present an approximation solution for the generalized maximum dynamic contraflow (GMDCF) problem using an FPTAS in Subsection 4.2. For the sake of completeness the earlier results to its solutions are stated in Subsection 4.1 . 


\subsection{Generalized maximum dynamic contraflow}

The generalized maximum dynamic contraflow problem is a maximum dynamic contraflow MDCF problem in which each arc contains capacity, transit time as well as loss factor. Considering a two terminal lossy network it is assumed that each arc has the proportional loss factor i.e., $\lambda \equiv 2^{c . \tau}$ for some constant $c<0$. It is also assumed that the loss per arc remains same in either direction.

Problem 3. Given a generalized network $\mathcal{N}=\left(V, A, b_{A}, \tau, \lambda, s, d, T\right)$, the GMDCF problem requires to find the maximum amount of flow that can be sent from $s$ to $d$ within time $T$ if the direction of the arcs can be reversed at time zero.

In order to solve Problem 3, Pyakurel et al. [14] and Pyakurel [13] presented an efficient generalized maximum dynamic contraflow (GMDCF) algorithm. Their GMDCF algorithm is based on the MDCF algorithm 22] for the MDCF problem and GMDF algorithm [7, 8, for the GMDF problem on two terminal lossy network. First, the given network is converted into auxiliary network using contraflow configuration where two-ways capacities are added to form new capacities, transit times remain same and loss factor depends upon the transit time. On auxiliary network, the GMDF algorithm of [7, 8] is used to compute GMDF optimally. It starts with the zero flow, computes a maximum flow in the minimum loss path, augments this flow and repeats this process until no $s-d$ path exists in the static residual network. Then, it uses the augmented maximum flows to construct an optimal solution by sending each flow as long as possible through the network similar to the temporally repeated flow technique for standard maximum dynamic flow. However, it takes pseudo-polynomial time to compute the GMDF on auxiliary network. Thus, the complexity of GMDCF algorithm of [13, 14] is pseudo-polynomial time. Their result is stated as follows.

Theorem 4.1. Every GMDF solution on auxiliary network is equivalent to the GMDCF solution on original network.

The minimum lost path is the successive shortest path from the source to the sink as $\lambda \equiv 2^{c \tau}$ for $c<0$ and there is always an optimal solution for the GMDCF problem on two terminal lossy network. The optimal solution induced by GMDCF algorithm of [13, 14 has the earliest arrival property, i.e., a cumulative amount of flows reaching the sink in every considered time period and all preceding time periods of the considered one have to be maximal. Thus, the generalized earliest arrival contraflow (GEACF) problem (cf. Problem 4h has been introduced and its solution has been obtained in pseudo-polynomial time iterations [13, 14.

Problem 4. For a generalized network $\mathcal{N}=\left(V, A, b_{A}, \tau, \lambda, s, d, T\right)$, the $G E A C F$ problem requires to compute the maximum flow that can be sent in every time period $0 \leq t \leq T$, from $s$ to $d$ with arc reversal capability at time zero.

\subsection{Approximate generalized maximum dynamic contraflow}

It is still unknown whether there exists a strongly polynomial time algorithm to solve the GMDCF problem. Even in the case without contraflow, there is no any polynomial time algorithm to solve the GMDF problem. We propose an FPTAS that computes an approximate GMDCF solution within time horizon $T$ in polynomial time complexity.

Problem 5. Given a generalized network $\mathcal{N}=\left(V, A, b_{A}, \tau, \lambda, s, d, T\right)$ and $\epsilon>0$. Does there exit an approximate GMDCF solution with value at least within a factor of $(1-\epsilon)$ of an optimal GMDCF solution within time horizon $T$ if the direction of the arcs can be reversed at time zero?

To solve Problem 5 we propose following algorithm (cf. Algorithm 3 ) that computes an approximate GMDCF solution. Step 2 constructs an auxiliary network by reversing the arcs at time zero. It is an undirected network in which capacity of each arc is increased but transit time remains same and loss factor depends on new transit time. Moreover, with increased capacity, the flow is also increased. In Step 3, we use the technique of FPTAS introduced in [7, 8, and compute an approximate generalized maximum dynamic flow on auxiliary lossy network. Note that an FPTAS is a fully polynomial time approximation scheme in which all approximation algorithms have a running time polynomial in the input size of the problem 
and $\epsilon^{-1}$. Authors in [7, 8, has obtained an FPTAS by terminating the GMDF algorithm after a polynomial number of iterations that approximates the maximum flow value rather than the required time horizon. Algorithm 3 gives an approximate solution early than the optimal solution to the GMDCF problem for two terminal lossy network. This algorithm turns into an FPTAS. There is no cycle with positive flow in Step 3 , thus Step 4 is well defined.

Algorithm 3. Approximate generalized maximum dynamic contraflow on lossy network

1. Input: Given the generalized network $\mathcal{N}=\left(V, A, b_{A}, \tau, \lambda, s, d, T\right), \epsilon>0$.

2. Construct the generalized auxiliary network $\overline{\mathcal{N}}=\left(V, E, b_{E}, \tau, \lambda, s, d, T\right), \epsilon>0$.

3. On $\overline{\mathcal{N}}$, compute an approximate generalized maximum dynamic flow with capacity $b_{E}(\bar{e})$, travel time $\tau(\bar{e})$ and the unique loss factor $\lambda(\bar{e}) \equiv 2^{\text {c. } \tau(\bar{e})}, c<0$ using an FPTAS of [7, 8].

4. Arc $(w, v) \in A$ is reversed, if and only if the flow along arc $(v, w)$ is greater than $b_{A}(v, w)$ or if there is a non-negative flow along arc $(v, w) \notin A$.

5. Output: An approximate GMDCF solution on two terminal lossy network $\mathcal{N}$.

Before proving the correctness of our, we prove that the technique of FPTAS introduced in [7, 8] computes an approximate generalized maximum dynamic flow in Step 3 with following lemma.

Lemma 4.2. For given $\epsilon>0$ and $U=\max _{e \in E} b_{E}(\bar{e})$, let $O P T$ be the value of an optimal GMDF solution on two terminal lossy auxiliary network $\overline{\mathcal{N}}$. Then on the same network, the technique of FPTAS obtains a GMDF solution of value

1. at least $O P T-\epsilon$, after all paths of length $\leq \tau^{*}$, where

$$
\tau^{*}=-\frac{1}{c}\left(\log \frac{1}{\epsilon}+\log m+\log U+2 \log T\right)
$$

2. at least $(1-\epsilon) O P T$, after $\tau^{*}$ iterations, where

$$
\tau^{*}=\left\lceil-\frac{1}{c}\left(\log \frac{1}{\epsilon}+\log m+\log U+2 \log T\right)\right\rceil
$$

Proof. First we prove the Step 1. We compute an optimal OPT GMDF solution using GMDF algorithm of [7, 8] on two terminal lossy auxiliary network $\overline{\mathcal{N}}$. Let $\omega, \omega \in\{0, \ldots, T\}$ be length (transit time) of a path and let $x_{\text {stat }_{\omega}}$ be the amount of flow sent into paths of length $\omega$ in a time step $t \in\{0, \ldots, T-\omega\}$ by the algorithm. The length of paths are monotonically increasing. There is no holdover so that the loss factor is $2^{c . \omega}$ and the flow is sent up to $T-\omega$ time steps through the path of length $\omega$. Suppose we have all paths of length $\leq \omega$. Then there remains only paths of length $\omega+1, \ldots, T$. Total flow reached to the sink using the remaining paths is

$$
\sum_{i=\omega+1}^{T} x_{\text {stat }_{i}} \cdot 2^{c . i} \cdot(T-i)
$$

For $c<0$, we have $\max \left\{2^{c(\omega+1)}, \ldots, 2^{c(T)}\right\}=2^{c(\omega+1)}$ and $T-i \leq T$. The total flow sent into the paths in one time step is bounded by $m . U$. This gives

$$
\sum_{i=\omega+1}^{T} x_{\text {stat }_{i}} \cdot 2^{c . i} \cdot(T-i) \leq \sum_{i=\omega+1}^{T} m \cdot U \cdot 2^{c(\omega+1)} \cdot T=m \cdot U \cdot 2^{c(\omega+1)} \cdot T^{2}
$$

If $\omega=-\frac{1}{c}\left(\log \frac{1}{\epsilon}+\log m+\log U+2 \log T\right)-1$ is assumed, then right hand side equals $\epsilon$.

Secondly, we prove the Step 2. As in the proof of Step 1, we assume that $\omega_{0}$ be the length of the paths in the first iteration. We assume that the network has the minimum capacity 1 . So that at least $2^{\text {c. } \omega_{0}}$ flow is sent in the first iteration. The problem is to find $\tau^{*}$ such that the flow amount reached to the sink by later iterations is $\leq \epsilon . O P T$. Then we obtain 


$$
\sum_{i=\omega_{0}+\tau^{*}}^{T} x_{s t a t_{i}} \cdot 2^{c . i} \cdot(T-i) \leq m . U \cdot 2^{c\left(\omega_{0}+\tau^{*}\right)} \cdot T^{2} \leq \epsilon 2^{c . \omega_{0}} \leq \epsilon . O P T
$$

We transform the Inequality 6 to

$$
m \cdot U \cdot 2^{c \tau^{*}} \cdot T^{2} \leq \epsilon \Leftrightarrow \tau^{*} \geq-\frac{1}{c}\left(\log \frac{1}{\epsilon}+\log m+\log U+2 \log T\right)
$$

Thus the proof is concluded.

Theorem 4.3. Algorithm 3 solves the approximate generalized maximum dynamic contraflow problem on two terminal lossy network optimally.

Proof. First of all we show that Algorithm 3 is feasible. In Step 2, we reverse the directions of arcs from sources to sinks so that capacity of each element containing on each arc is added to form new capacity. According to contraflow configuration, transit time and loss factor does not influence the feasibility. So that Step 2 is feasible. As the FPTAS introduced by 7,8 is feasible, Step 3 is feasible. In Step 3, there is no any cycle with positive flows so that flow in Step 4 is either along $\operatorname{arc}(v, w)$ or $(w, v)$ but never in both directions at the same time as well as at different time periods. As a result, the flow is not greater than the reversed capacities on all the arcs at all time periods. Thus, Step 4 is also feasible.

Now the optimality of the algorithm is shown as follows. From the feasibility condition, we have that every feasible approximate generalized maximum dynamic flow in the lossy auxiliary network $\overline{\mathcal{N}}$ is feasible to the approximate generalized maximum dynamic contraflow solution in the lossy network $\mathcal{N}$. Lemma 4.2 obtains an optimal approximate GMDF in two terminal lossy auxiliary network. As the amount of flow obtained in Step 3 cannot be changed in Step 4, the resulting flow is an optimal solution to the approximate generalized maximum dynamic contraflow problem. Thus, by Theorem 4.1, the approximate GMDF solution in auxiliary network $\overline{\mathcal{N}}$ is equal to the approximate GMDCF solution on original network $\mathcal{N}$. This completes the proof.

Corollary 3. For given $\epsilon>0, U=\max _{e \in E} b_{E}(e)$, constant $c<0$ on $\mathcal{N}$, the Algorithm 3 solves the approximate GMDCF problem in $O\left(\max f \operatorname{low} \cdot\left(\log \epsilon^{-1}+\log U+\log T\right)\right)$ time complexity.

Notice that the special loss function on the arcs is the key to our FPTAS for the GMDCF problem. As there is neither a polynomial-time nor a polynomial-time value-approximation algorithm for the GMDF problem with arbitrary and lossy loss, unless $\mathbf{P}=\mathcal{N} \mathcal{P}[7,8$, this FPTAS fails to solve the GMDCF problem with arbitrary and lossy loss.

\section{Conclusions}

The GMSF problem and the GMDF problem have been studied in the literature. Likewise the analytical solution of static and dynamic contraflow problems have been studied. We combined both approaches on two terminal lossy network and introduced the GMSCF and the approximate GMDCF problems. We solved the GMSCF with efficient solution procedure on two terminal lossy network. As it has pseudo-polynomial time complexity, we computed its approximation solution on two terminal lossy network in polynomial time complexity. We also claimed that there exists an exact GMSCF solution with very small error scaling on two terminal lossy network even with flow generating cycle. Moreover, we presented an approximation solution for the GMDCF problem on two terminal lossy network. To the best of our knowledge, these problems we introduced in discrete time setting are for the first time in generalized evacuation planning.

The flow values obtained by these solutions increase significantly although our extended solution on contraflow has similar complexity of algorithm without contraflow. From existing literature, it is shown that the flow value may be doubled for a given time and time required to transship the given value can be two times faster in contraflow than without contraflow. We conjecture that if the dynamic cut capacities 
are symmetric and loss factor is proportional to the time, then the lossy networks compute double flow value for given time horizon with contraflow configuration.

Krumke and Zeck [10] solved the generalized maximum static flow problem using the greedy-strategy on two-terminal series parallel graph. We conjecture that an optimal solution for the GMSCF problem on two-terminal series-parallel graph can be obtained efficiently using the greedy-strategy on generalized maximum auxiliary network that chooses minimum lost path in original network. However it does not works in residual network. We also conjecture that greedy-strategy can be carried over to our setting and can be used for bounding the number of paths used for GMDCF and GEACF problems. Since each augmentation saturates an arc, there can be at most $m$ iterations, yielding a polynomial time algorithm.

Acknowledgments: The author acknowledges the partial support of DAAD under the partnership program between the departments of mathematics at Tribhuvan University, Nepal, Mindanao State UniversityIIT, Philippines and University of Kaiserslautern, Germany, entitled Graph Theory and Optimization with Applications in Industry and Society (GraTho). The author also thanks the support of Alexander von Humboldt Foundation under the AvH Research Group Linkage Program at TU Bergakedemie Freiberg (August 18-November 14, 2016). The authors would like to thank the anonymous referees and the editor for their valuable suggestions to improve the quality of this paper.

\section{References}

[1] Arulselvan A (2009) Network model for disaster management. PhD Thesis, University of Florida, USA.

[2] Dhamala TN (2015) A survey on models and algorithms for discrete evacuation planning network problems. Journal of Industrial and Management Optimization, 11:265-289.

[3] Dhamala TN and Pyakurel U (2013) Earliest arrival contraflow problem on series-parallel graphs. International Journal of Operations Research, 10:1-13.

[4] Dhamala TN and Pyakurel U (2016) Significance of transportation network models in emergency planning of urban cities. International Journal of Cities, People and Places, 2:58-76.

[5] Fleischer LK and Wayne KD (2002) Fast and simple approximation schemes for generalized flow. Mathematical programming, 91:215-238.

[6] Goldberg AV, Plotkin SA and Tardos E (1991) Combinatorial algorithms for the generalized circulation problems. Mathematics of Operations Research, 16:351-381.

[7] Gross M (2014) Approximation algorithms for complex network flow over time problems. PhD Thesis, Technical University, Berlin, Germany.

[8] Gross M and Skutella M (2012) Generalized maximum flows over time. Proceedings of the $9^{\text {th }}$ Workshop on Approximation and Online Algorithms, Lecture Notes in Computer Science, 7164:247-260.

[9] Kim S, Shekhar S and Min M (2008) Contraflow transportation network reconfiguration for evacuation route planning. IEEE Transactions on Knowledge and Data Engineering, 20:1-15.

[10] Krumke SO and Zeck C (2013) Generalized max flow in series-parallel graphs. Discrete Optimization, 10:155-162.

[11] Onaga K (1966) Dynamic programming of optimum flows in lossy communication nets. IEEE Transactions on Circuit Theory, 13:282-287.

[12] Onaga K (1967) Optimal flows in general communication networks. Journal of the Franklin Institute, 283:308327.

[13] Pyakurel U (2016) Evacuation planning problem with contraflow approach. PhD Thesis, IOST, Tribhuvan University, Nepal. 
[14] Pyakurel U, Hamacher HW and Dhamala TN (2014) Generalized maximum dynamic contraflow on lossy network. International Journal of Operations Research, Nepal, 3:27-44.

[15] Pyakurel U and Dhamala TN (2014) Earliest arrival contraflow model for evacuation planning. Neural, Parallel, and Scientific Computations, 22, 287-294.

[16] Pyakurel U and Dhamala TN (2015) Models and algorithms on contraflow evacuation planning network problems. International Journal of Operations Research, 12:36-46.

[17] Pyakurel U and Dhamala TN (2017) Evacuation planning by earliest arrival contraflow. Journal of Industrial and Management Optimization, 13:487-501.

[18] Pyakurel U and Dhamala TN (2016a) Continuous time dynamic contraflow models and algorithms. Advance in Operations Research; Article ID 368587, 1-7.

[19] Pyakurel U and Dhamala TN (2017) Continuous dynamic contraflow approach for evacuation planning. Annals of Operation Research, 253:573-598.

[20] Pyakurel U, Dhamala TN and Dempe S (2017) Efficient continuous contraflow algorithms for evacuation planning problems. Annals of Operations Research (ANOR), 254:335-364.

[21] Pyakurel U, Goerigk M, Dhamala TN and Hamacher HW (2016) Transit dependent evacuation planning for Kathmandu valley: A case study, International Journal of Operations Research Nepal, 5(1): 49-73.

[22] Rebennack S, Arulselvan A, Elefteriadou L and Pardalos PM (2010) Complexity analysis of maximum flow problem with arc reversal. Journal of Combinatorial Optimization, 29:200-216.

[23] Tardos E and Wayne KD (1998) Simple generalized maximum flow algorithms. Integer Programming and Combinatorial Optimization, Lecture Notes in Computer Science, 1412:310-324. 